\title{
2 \\ Interstellar Songwriting: What Propels a Song Beyond Escape Velocity?
}

\author{
Clive Harrison
}

\section{Introduction}

Within the broadest cultural realm, and through the varied creativity definitions and process models presented by scholars (Bastick, 1982; Csikszentmihalyi, 1997, p. 80; Cropley, in Kaufman and Beghetto, 2009; Rothenberg and Hausman, 1976, p. 14; Sawyer, 2006, p. 89; Wallas, 1926), songwriting resides as a small but influential sub-domain of popular culture. Kaufman and Beghetto (2009) proposed an expanded model of creative magnitude in which mini-c refers to creativity in children, little-c refers to everyday innovation, Pro-c refers to professional creative expertise and Big-C refers to eminent accomplishment of paradigmshifting or domain-changing creativity (pp. 1-12). The research question can be expressed in the following terms: "how can the professional (Pro-c) songwriter move from "planetary" creativity, to "interstellar" (paradigmshifting) Big-C creativity?. Rather than view the songwriting goal as a binary, such as exploration and process, or the presentation of an artistic product, it is suggested that it is a confluence of all three. That is, a willingness and enthusiasm for exploration, a fascination with process and a consciousness of the presentation of an artistic product. 
From McIntyre's (2003, p. 228) synthesis of theorists Csikszentmihalyi, Bourdieu and Negus, the most valuable lessons in this discussion for the songwriter are the following perspectives:

- Simply writing songs is necessary but not sufficient.

- Distribution and access to the domain and the field are vital.

- We write songs having first absorbed the song culture of the time.

- We cannot avoid having been influenced in our songwriting decisions in a profound way.

Also significant is the notion that for a song to become part of the domain it must be deemed worthy by the filter of the music industry (Csikszentmihalyi's field), and that songwriters act within a songwriting system of creativity in which agency and structure are interdependent. Further, Becker's Art Worlds (1982) reminds us that within the songrecording world, engineers, producers and other creative agents contribute to the song outcome and its potential for inclusion in the domain.

To consistently deliver worthy song contributions to the songwriting domain as a field professional, the Pro-c songwriter(s) need to act within the systems model of creativity (Csikszentmihalyi, 1988; Feldman, Csikszentmihalyi and Gardner, 1994). That is, they must acquire an expert knowledge of the domain, create songs that are deemed creative (for simplicity, defined herein as novel, useful and nonobvious) ${ }^{1}$ and have access to the field - the experts, intermediaries, gatekeepers, critics and audience in that society.

1 Over the past 25 years, researchers have attempted to fine-tune a definition of creativity through a range of terminologies. For example, Kaufman and Sternberg (2010) vouch for the inclusion of 'originality', and highlight 'novelty' and 'quality', where quality also implies 'good' or 'useful' (p. 467). Gardner (1993) adds 'solving problems' and 'defining new questions' as outcomes of creativity (p. 33), and (noting the inculcation of habitus as described by Bourdieu) McIntyre (2006) includes notions of 'antecedent conditions' and makes the distinction of adding to the knowledge. Boden (2012) includes the term 'surprising' (p. 12), Weisberg (1993) requires the production of 'goal-directed novelty' (p. 244) and Sawyer (2006) advocates 'appropriateness' in his sociocultural definition of the term (p. 9). In deference to the United States Patent Office, Dean Keith Simonton (2014) includes the terms 'original' and 'non-obvious', the implication being that the invention would not be considered obvious to someone who has 'ordinary skill in the art'. 


\section{Overview}

For this discussion, songwriting will be viewed as a creative task with varying degrees of magnitude from mini-c, through little-c, then Pro-c and, finally, the Big-C creativity of exemplars. Located within the sociocultural, and subject to the interdependency of field and domain, the following factors identified as influencing the creative process are examined: discriminant pattern recognition, naturalistic intelligence, productivity, fruitful asynchrony, propulsion theory, risk, field switching, expert variation and selective retention, and the production of significant works. A new term, 'adeptus', is offered to describe the confluence of these factors. For songwriters, it is posited that focus should be directed to its acquisition, rather than to factors beyond their control (the opinions of a particular music critic, for example, or a fortuitous opportunity occurring).

\section{Adeptus and its Acquisition}

By acquiring the habitus (Bourdieu, 1983) of the songwriting domainthat is, knowledge of the practical skills, techniques, concepts and procedures necessary for creating appropriate lyric, melody, harmony, rhythm, texture and style - quality song artefacts are more likely to be consistently created. Directed practice and reflection on songwriting procedures over an extended period is also necessary to develop expertise as a songwriter (as is the case with instrumental practice). Knowledge of the songwriting symbol system - the language, terminology and notation of popular music - is not essential, but is certainly an advantage for controlling performance elements and degrees of player interpretation on the live stage and in the studio. By acquiring such knowledge, quality song artefacts are more likely to be consistently created. The specific nomenclature and language of songwriters is geographically and historically inconsistent, changing generationally with conversational speech. As a result, it behoves songwriters to establish a common and contemporary argot for use, discussion, interaction and education. For the contemporary songwriter, however, domain knowledge from formal tuition alone is insufficient. 
Songwriters benefit from, and need to be cognisant of, the role of informal, self-directed education and the vital domain acquisition derived beyond the classroom: the tacit knowledge and expertise of the seasoned professional. When habitus (through deep domain immersion), tacit knowledge (Polanyi, 1966; Schön, 1983), intuition (nonlinear parallel processing of global multicategorised information) (Bastick, 1982) and the unique distinctions borne of discriminant pattern recognition (naturalistic capacity) (Gardner, 1999) are combined with recursive, directed practice and reflection over an extended period, the resultant expertise or 'adeptus' (Harrison, 2016a) is likely to move creativity from little-c to Pro-c.

Adept: expert, skilful, nimble-fingered, capable, polished, professional, masterful.

Adeptus: all the attributes of an expert or master. More precisely:

The combined expertise and tacit acquired knowledge resulting from the confluence of habitus (through deep domain immersion), intuition (nonlinear parallel processing of global multi categorised information), the unique distinctions borne of discriminant pattern recognition (naturalistic capacity) and the application of recursive, directed practice and reflection over an extended time-period. (Harrison, 2016a, pp. 396-97)

To move beyond Pro-c into the realm of Big-C creativity (cognisant that this is an attribution given 'after-the-fact' by the field of experts), it is argued that facilitative factors include a fruitfully asynchronous perspective, the capacity to challenge or reject the current paradigm and a willingness to risk rejection. It is not asserted that these elements provide all that is necessary for Big-C creativity to occur, merely that they are necessary for exemplary songwriting.

\section{Discriminant Pattern Recognition}

It is posited that an exceptional capacity for discriminant pattern recognition - that is, recognising, identifying and responding to important stimuli from the songwriting domain-is correlated to the Big-C creativity of exemplars. The very best songwriters simply observe in greater detail and respond more accurately to the culture in which they work creatively. Their unique perspective, experience and adeptus (all the attributes of the expert or master) provide the skill required to make nuanced choices that are 
otherwise unavailable to the average songwriter. Highly desirable for the songwriter, then, would be a strong capacity for naturalistic intelligence (Gardner, 1999) in the sense of its relation to song evolution and species (Bennett, 2011, 2012; Perkins, in Boden, 1996, p. 126).

\section{Naturalistic Intelligence}

In his multiple intelligence (MI) theory, Gardner (1983) presents a useful tool for songwriting practitioners that is especially geared to redress some of the imbalance of the general intelligence- or 'g'-based education system favoured by Spearman (1987, pp. 201-292). His 'eight intelligences' view (Gardner, 1999) provides a useful construct for examining creativity, and specifically songwriting, within the cultural milieu. ${ }^{2}$ Prior research in the field has not specifically addressed songwriting practice. However, recent research (Harrison, 2016a), has uncovered the following distinctions.

At various stages in the songwriting process, songwriters may use all eight MIs: musical-aural and linguistic-verbal skills; the logicalmathematical skills of chord theory; recording and composition; visualspatial factors in the recording of the song artefact using architectural, aural and temporal space; bodily-kinesthetic instrumental and/or vocal performance skills; and well-developed interpersonal, intrapersonal and naturalistic capacities. The last three capacities are highly valued and more likely to be selected by the field for their ability to reflect, specifically and consistently, what is valued, novel and nonobvious to the audience. Curiously, on this researcher's first view of MI theory some years ago, the capacity for naturalistic intelligence, described in Darwinian/evolutionary terms (Gardner, 1999, p. 48), seemed remote and possibly irrelevant to songwriting research. However, it can now be perceived as highly valuable-reflecting the capacity of songwriters to correctly identify and reproduce accurate song 'species'. A Darwinesque survival of the fittest is at play (Bennett, 2012), where songs survive or become extinct based upon whether the 'field' accepts the song artefact as a worthy inclusion in the domain. For recursive artefact evaluation during the creative process, astute use of naturalistic skill is a powerful differentiator between moderate and outstanding songwriting outcomes.

2 It should be noted that multiple intelligence theory takes a cognitive view of creativity, and is based on the individual node as situated within the systems model (Csikszentmihalyi, 1988; Feldman, Csikszentmihalyi and Gardner, 1994) that includes two other nodes, domain and field. 
Significantly, this form of naturalistic domain acquisition is not normally included in formal music education. Its development relies largely on selfdirected, informal immersion in the subculture (Green, 2013), and is most often achieved in two ways: by prolific songwriting, testing those songs on audiences, observing reactions and writing new songs for subsequent testing; or, through studious directed listening to songs that have been already accepted into the domain as worthy by the field, observing what appears to work and considering potential generalities. Both require the songwriter to actively participate outside the confines of the studio or the classroom-in the field itself.

Songwriting based upon deep immersion in a specific song style tends to garner industry support. Embracing the forward incrementation proposed by Sternberg, Kaufman and Pretz (2002), when incremental (rather than radical) change is preferred in creative practices in which the field is content with the state of the domain, the field recognises the near-analogous nature of the song compared with its antecedents. Song artefacts whose lineage and style are clear, then, tend to be more popular in commercial markets than those that are not. Conversely, artefacts whose lineage and style are obscure are more popular in avant-garde and local markets, as described by Lena and Peterson (2008), than those whose target market is obvious. However, for Thistlethwayte (2015), a heightened awareness of song lineage and antecedents is common, but it is desirable to use those earlier works as inspiration for exploration, rather than merely copying. The experienced professional can deconstruct his or her works post-hoc, and recognise influences (Connors, 2015), but what is valued is the unaccountable and personal choices they have made based on their own adeptus.

\section{Productivity}

For Sawyer (2006, p. 131), the popular notion of the solitary creator working on a single masterpiece that will change the world is 'more of a myth than reality'. Domain-changing or significant creativity is more commonly the realm of the prolific, highly productive, persistent agentthe creator of multiple works whose dogged persistence bears fruit (Gardner, 1993, pp. 343-345). Inventors and creators whose work is judged to be truly significant over time have the highest overall lifetime output, be they inventors (Huber, 1998, pp. 58-72) or creators (Simonton, 
1988a, 1988b). Sawyer (2006, p. 131) describes such a creative process as the productivity theory, arguing that 'the best way to have a good idea is to have a lot of ideas, and then just get rid of the bad ones'. This suggests that by creating multiple artefacts, inventors and creators maximise their chance for an artefact to be deemed novel, useful and nonobvious (i.e. creative). The implication for Pro-c songwriters is that the goal is not to attempt to produce a singular masterpiece; rather, it is to produce a body of songwriting works from which the field may potentially select one or more masterpieces.

A further distinction should be made regarding high productivity. While the production of truly significant works can be correlated with highly productive creators, Sawyer's notion does not insist that such truly significant works must also constitute domain-changing or paradigmshifting works. They may simply be deemed significant-that is, the best version, product or idea in the domain, based upon the collective evaluation of the field. For the Pro-c creative songwriter who does not necessarily set out to change the domain, and whose works follow the forward incrementation of someone content with the state of the domain and has no need to shift it, such an attribution is entirely possible and available.

What then of the highly productive, expert Pro-c songwriter who contributes multiple significant works over a lifetime but falls short of the ideal of paradigm-shifting creativity? For example, some might argue that the songs of Grammy award-winning Swedish producer and expert songwriter Max Martin have not shifted the domain of mainstream pop per se. However, it would be hard to argue that he has not produced some significant works among his 54 top-10 hits and 19 US number ones. From the perspective of Sternberg, Kaufman and Pretz's (2001) propulsion theory, his writing style is more accepting of the current paradigm, rather than rejecting or challenging it. While Martin's Grammy tally approaches that of John Lennon, some may assert that Lennon was the more creative; although to address that debate one would have to delve into sociocultural notions of authenticity beyond the scope of this chapter. From this research perspective, Lennon was willing to challenge or reject aspects of the existing paradigm, whereas Martin seems to have been content to accept and extend it. So, the question becomes: what provides the necessary extra impetus or propulsive power to propel the songwriter beyond functional Pro-c songs to eminent, significant or domain-changing Big-C creativity? 


\section{Fruitful Asynchrony}

Howard Gardner (1993, p. 353) describes the distinction between factors influencing those who become 'creative' beyond mere 'experts', as fruitful asynchrony-that is, the 'capacity to exploit, or profit from, an apparent misfit or lack of smooth connections within the triangle of creativity'. This 'triangle of creativity' refers to the individual, domain and field of Csikszentmihalyi's systems model of creativity (1988). Applied to songwriting, asynchrony occurs when a songwriter is in some way out-ofstep with the song culture of the day. That asynchrony becomes fruitful when it leads to the type of song that shifts the paradigm of the prevailing songwriting landscape, the realm of the Big-C creative songwriter. Those asynchronously fruitful songs can be described in terms of differently conceived creative propulsions.

\section{Propulsion Theory}

While a proliferation of songs, adequate expertise, creativity and access to the field might push the songwriter to a Pro-c level, creating songs that change the domain or shift the paradigm requires some additional (or alternative) skills. To actively change or shift the existing culture, it would be helpful at first to have perceived a need for change, or to be dissatisfied in some way with its current state. To that end, a perspective that is slightly different or fruitfully asynchronous with other songwriters would be desirable, along with an attendant willingness to create songs that challenge the current paradigms and potentially risk failure, or rejection. If each song is considered a creative propulsion, three types of new song paradigms are created: those that accept or extend the paradigm, those that reject or replace the current paradigm, and those that integrate or synthesise song styles. Further, eight types of creative propulsion are defined: replication, redefinition, forward incrementation, advance forward incrementation (that accept and/or extend), redirection, reconstruction, reinitiation (that reject or challenge) and synthesis (that integrates). 


\section{Risk}

Most common among propulsions in the songwriting realm is forward incrementation, where the songwriter attempts to propel forward the new style with another song artefact with similar forward momentum of style. The 'riskier' approach of attempting to reject or replace the current paradigm might lead to songs more likely to change the paradigm, but may be avoided by a songwriter whose desired outcome is cultural acceptance, rather than cultural notoriety or change. For the Pro-c songwriter who is averse to risk-taking (less enthusiastic about exploration) in their professional creative work, asynchronous song possibilities may be difficult or present as undesirable choices. However, Pro-c songwriters who see the exploratory aspects of songwriting as desirable and a welcome challenge rather than a risky constraint may find themselves moving into the realm of domain-changing and paradigm-shifting song artefacts. An inexperienced songwriter could blindly experiment with all sorts of ill-informed, wild song explorations searching for asynchronous iterations that might prove fruitful, but the expert, with the adeptus to make astute variations, is far more likely to discover those divergent, remotely analogous possibilities that will bear fruit. One factor contributing to the capacity to identify remotely analogous, rather than near-analogous song possibilities, is when creativity-supporting skills are transferred from other disciplines, as is sometimes the case with field-switchers.

\section{Field Switching}

Following Sawyer's (2006, p. 115) argument that, 'creative combinations often result when people switch fields', it is suggested that crossdisciplinary skills can provide a useful source of fruitful asynchrony that opens remotely analogical possibilities for the creative agent (Harrison, 2016b). When the expert is able to transfer expertise from a different field, their unique perspective may identify paradigms they feel warrant challenging or rejecting. If a polymath (with expertise across two different fields of endeavour) manages to transfer domain-specific or domain-general skills across disciplines, the resultant creative products or propulsions are likely to be different. In terms of propulsion theory, these redirections, reconstructions, re-initiations or syntheses move away from where we are now. Therefore, skills possessed across unrelated disciplines may precipitate more divergent, remote-analogical solutions than those 
convergent solutions implied by closely related, domain-specific transfers. Field switching may be a factor that stimulates the creative person to explore propulsions that move away from where we are now, by introducing the type of fruitfully asynchronous, remotely analogous ideas that (if accepted by the field as worthy) tend to shift the paradigm (Harrison, 2016b).

\section{Expert Variation and Selective Retention}

It is suggested here that the capacity to be creative (that is, to come up with valuable, useful and nonobvious solutions) is available to anyone. Additionally, 'highly creative' people simply direct their attention towards remote-analogies more freely and successfully than 'less creative' people, who, for one reason or another, seek near-analogies as a matter of habit. A willingness to test, explore and embrace blind variation and selective retention (BVSR) (Campbell, 1960) is implied, where song possibilities include trial and error, randomised choices, unlikely solutions to songwriting 'problems' and risk-taking. However, the songwriter who possesses outstanding adeptus - expertise, tacit knowing, discriminant pattern recognition and habitus-is capable of informed trial and error, nuanced choice-making, well-advised solutions and measured risk-taking, significantly more than the novice or moderately equipped songwriter. Following Weisberg, who proposes a 'continuum between expert-based creativity and BVSR, with the important dimension being the depth of knowledge that the individual brings to the situation' (as summarised in Hass and Weisberg, 2015, p. 471), it follows that a highly skilled, deeply immersed songwriter might apply his or her unique adeptus as a form of 'expert' variation and selective retention (EVSR), a form of discriminant pattern recognition unavailable to lesser songwriters. From this informed EVSR perspective, solution-seeking is much more likely to be successful, resulting in more song candidates for potential acceptance by the field as 'significant' works rather than the random choices of a novice whose variations are 'blind'.

\section{Significant Works}

Sawyer (2006, p. 131) has identified that truly significant works tend to come from highly productive creators, but it is posited here that the distinction should be made that not all truly significant works are 
necessarily domain changing or paradigm shifting. Some may be simply the stand-out version, product or idea in that domain at the time. Until the field has cast its vote, we won't know which works are going to change the domain, and that may take years, decades or even centuries. Describing creativity that is 'new in history', Boden (1991, p. 269) states, 'what we identify as "H-creative" depends to a large extent on historical accident and social fashion'. This situation raises the songwriting question as to whether the attempt to create 'significant works' necessarily includes the requirement to change the domain or shift the paradigm.

Further, the question 'how can the songwriter move from (Pro-c) creativity, to (domain-changing) Big-C creativity?' is revealed to be misleading. It implies that the goal of the songwriter should be to change the domain, despite it having been shown that Big-C creativity is an attribution by the field, after the fact, as described by Sawyer (2006, p. 124): 'a creation's ultimate social importance can't be predicted from the mental processes involved; it results from a social process'. We can view it simply as a domainchanging iteration of Pro-c creativity. Thus, it is posited that the following distinction describes more accurately the songwriting continuum of 'greatness'. Within the broad description of Pro-c songwriting exists two forms of exemplar creativities: Big-C creativity includes all significant or eminent creativities whatever the style of creative propulsion, while a new term, 'Alt-C' creativity, includes 'alternative' creative propulsions that have been observed by the field to shift the paradigm or change the domain.

\section{Conclusions}

The confluent application of a songwriting methodology that embraces the systems model of creativity (Csikszentmihalyi, 1988), maximises the capacities of available MI (Gardner, 1983) and exploits creative propulsions acceptable to the field (Sternberg et al., 2002) is likely to be highly productive. Further, it is desirable to acquire a high level of expertise, allowing distinctions to be made that others would miss, or fail to recognise, as significant. These creators will make exceptional choices based on 'intuition' (i.e. 'feelings of warmth') (Nickerson, in Sternberg, 1999) as to the solutions most likely to produce novel and useful variations within the domain. They will apply the dogged persistence, determination, focus, application and other attributes described as qualities of the creative personality (Boden, 1996; Csikszentmihalyi and Csikszentmihalyi, 
1991; Gardner, 1993) to arrive at the necessary adeptus of the expert or master. Supporting Boden's (1996) view of creativity, where H (historical) creativity is a subset of $\mathrm{P}$ (personal) creativity, it is posited that both Big-C and Alt-C creative songwriting can be considered subsets of Pro-c creativity (Harrison, 2016b) - the former representing significant song artefacts that accept or extend the paradigm (incrementally moving it forward), and the latter representing significant song artefacts that reject or challenge it (shifting the paradigm). The question as to 'what factors move the songwriter along the continuum from Pro-c creativity into the realm of Big-C creativity' no longer necessarily implies a hierarchy in which the highest goal of the songwriter should be to change the domain, and that Big-C is somehow greater than excellent Pro-c creativity. Rather, Big-C (and Alt-C) creativity can be considered significant iterations of Pro-c songwriting craft.

To move from planetary to interstellar songwriting, six contributory facilities have been identified. The first three precipitate the shift from Pro-c to Big-C creativity. Discriminant pattern recognition is the facility, in an evolutionary sense, to recognise similar species across domains and possess the adequate powers of differentiation, observation and evaluation to make informed distinctions that others cannot see (EVSR). Metacognition, Big-C creativity, requires a capacity for higher-order thinking skills that include analysis, evaluation and creation, and the resources (including time) to test for validity and reliability. Finally, productivity involves creating multiple artefacts to maximise the chances that one or more will be deemed novel and useful (i.e. creative).

Three further contributory facilities specifically direct creative outputs towards domain-changing or paradigm-shifting Alt-C creativity. Fruitful asynchrony is an asynchronous perspective that challenges the current paradigms. To be Alt-C creative, one would need to make distinctions differently to others in the domain to be able to create differently. Fieldswitching - expertise in multiple disciplines—is a fruitfully asynchronous factor that stimulates the creative person to explore propulsions that move away from the current position of the field. Field-switchers are more likely to make distinctions that other, less expert, less interdisciplined people cannot. Finally, risk is a willingness to explore possibilities without fear of failure. A successful Pro-c songwriter might be simply unwilling to take paradigm-challenging risks, and seek instead to create significant works that do not change the domain. 
The idiosyncratic depth perspective and adeptus of the significant, interstellar Big-C songwriter utilises a capacity to observe and apply nuances of style, technique and habitus unnoticed or missed completely by the lesser songwriter. The very best songwriters, it would seem, simply listen better, notice differently and document their unique perspectives in song based on higher levels of adeptus.

\section{References}

Bastick, T. 1982. Intuition: How We Think and Act. Hoboken: John Wiley \& Sons.

Becker, H. 1982. Art Worlds. Berkeley: University of California Press.

Bennett, J. 2011. 'Collaborative Songwriting - The Ontology of Negotiated Creativity in Popular Music Studio Practice'. Journal on the Art of Record Production 5. arpjournal.com/collaborative-songwriting---theontology-of-negotiated-creativity-in-popular-music-studio-practice/ (accessed 10 May 2018).

- 2012. 'The Song Remains the Same-Why?' Total Guitar, June, 228. joebennett.net/2013/02/11/the-song-remains-the-samewhy-from-total-guitar-magazine/ (accessed 10 May 2018).

Boden, M. 1991. The Creative Mind: Myths \& Mechanisms. New York: Basic Books.

—_. 1996. Dimensions of Creativity. Cambridge: MIT Press.

—_. 2012. Creativity and Art: Three Roads to Surprise. Oxford: Oxford University Press.

Bourdieu, P. 1983. The Field of Cultural Production: Essays on Art and Literature. New York: Columbia University Press.

Campbell, D. T. 1960. 'Blind Variation and Selective Retentions in Creative Thought as in Other Knowledge Processes'. Psychological Review 67 (6): 380. doi.org/10.1037/h0040373

Connors, G. 2015. Interview by author. Mackay, QLD. 28 August. 
Csikszentmihalyi, M. 1988. 'Society, Culture, and Person: A Systems View of Creativity'. In Conception of Giftedness, edited by R. J. Sternberg and J. Davidson, 25-339. New York: Cambridge University Press.

- 1997. Finding Flow: The Psychology of Engagement with Everyday Life. New York: Basic Books.

Csikszentmihalyi, M. and I. S. Csikszentmihalyi. 1991. Flow: The Psychology of Optimal Experience, Vol. 41. New York: Harper Perennial.

Feldman, D. H., M. Csikszentmihalyi and H. Gardner. 1994. Changing the World: A Framework for the Study of Creativity. Westport: Praeger.

Gardner, H. 1983. Frames of Mind - The Theory of Multiple Intelligences. New York: Basic Books.

- 1993. Creating Minds: An Anatomy of Creativity as Seen Through the Lives of Freud, Einstein, Picasso, Stravinsky, Eliot, Graham, and Gandhi. New York: Basic Books.

- 1999. Intelligence Reframed: Multiple Intelligences for the 21st Century. New York: Basic Books.

Green, P. L. 2013. How Popular Musicians Learn: A Way Ahead for Music Education. Farnham: Ashgate Publishing.

Harrison, C. M. 2016a. 'A Songwriter's Journey from Little-c to Pro-C Creativity: An Applied Analytical Autoethnography'. PhD thesis, University of Newcastle, Newcastle, NSW.

— 2016b. 'Bebop on the Hockey Pitch: Cross-Disciplinary Creativity and Skills Transfer'. Performance Science 7: 123. doi.org/10.3389/ fpsyg.2016.00123

Hass, R. W. and R. W. Weisberg. 2015. 'Revisiting the 10-Year Rule for Composers. From the Great American Songbook: On the Validity of Two Measures of Creative Production'. Psychology of Aesthetics, Creativity, and the Arts 9 (4): 471-479. doi.org/10.1037/aca0000021

Huber, J. 1998. 'Invention and Inventivity as a Special Kind of Creativity, With Implications for General Creativity'. Journal of Creative Behavior 32 (1): 58-72. doi.org/10.1002/j.2162-6057.1998.tb00806.x 
Kaufman, J. C. and R. A. Beghetto. 2009. 'Beyond Big and Little: The Four-c Model of Creativity'. Review of General Psychology 13 (1): 1-12. doi.org/10.1037/a0013688

Kaufman, J. C. and R. J. Sternberg. 2010. The Cambridge Handbook of Creativity. Cambridge: Cambridge University Press. doi.org/10.1017/ CBO9780511763205

Lena, J. C. and R. A. Peterson. 2008. 'Classification as Culture: Types and Trajectories of Music Genres'. American Sociological Review 73 (5): 697-718. doi.org/10.1177/000312240807300501

McIntyre, P. 2003. 'Creativity and Cultural Production: A Study of Contemporary Western Popular Music Songwriting'. PhD thesis, Macquarie University, Sydney, Australia.

—_. 2006. 'Paul McCartney and the Creation of "Yesterday": The Systems Model in Operation'. Popular Music 25 (2): 201-219. doi.org/10.1017/S0261143006000936

Polanyi, M. 1966. The Tacit Dimension. Chicago: University of Chicago Press.

Rothenberg, A. and C. Hausman. 1976. The Creativity Question. Durham: Duke University Press.

Sawyer, R. K. 2006. Explaining Creativity: The Science of Human Innovation. Oxford: Oxford University Press.

Schön, D. 1983. The Reflective Practitioner: How Professionals Think in Action. New York: Basic Books.

Simonton, D. K. 1988a. 'Creativity, Leadership, and Chance'. In The Nature of Creativity: Contemporary Psychological Perspectives, edited by R. J. Sternberg, 386-426. New York: Cambridge University Press.

—_. 1988b. Scientific Genius: A Psychology of Science. Cambridge: Cambridge University Press.

—_. 2014. 'Thomas Edison's Creative Career: Multilayered Trajectory of Trials, Errors, Failures, and Triumphs'. Psychology of Aesthetics, Creativity, and the Arts 9 (1): 2-14. doi.org/10.1037/a0037722 
Spearman, C. 1987. 'The Proof and Measurement of Association between Two Things' (originally published 1904). The American Journal of Psychology 100 (3-4): 441-471. doi.org/10.2307/1422689

Sternberg, R. J. (ed.). 1999. Handbook of Creativity. Cambridge: Cambridge University Press.

Sternberg, R. J., J. C. Kaufman and J. E. Pretz. 2001. 'The Propulsion Model of Creative Contributions Applied to the Arts and Letters'. The Journal of Creative Behavior 35 (2): 75-101. doi.org/10.1002/j.2162-6057. 2001.tb01223.x

- 2002. The Creativity Conundrum: A Propulsion Model of Kinds of Creative Contributions. London: Routledge.

Thistlethwayte, R. 2015. Interview by author. Redfern, NSW. 27 April.

Wallas, M. G. 1926. The Art of Thought. London: C. A. Watts.

Weisberg, R. W. 1993. Creativity: Beyond the Myth of Genius. London: W. H. Freeman \& Company. 
This text is taken from Popular Music, Stars and Stardom, edited by Stephen Loy, Julie Rickwood and Samantha Bennett, published 2018 by ANU Press, The Australian National University, Canberra, Australia.

doi.org/10.22459/PMSS.06.2018.02 